\title{
Reduced-size Microstrip Four-Pole Bandpass Filter Using Two-layer Structure with Quarter-Wavelength Resonators and Open Stub Inverters
}

\author{
Azzeddine Djaiz and Tayeb A. Denidni and Halim Boutayeb \\ INRS-EMT, University of Québec, 800 rue de la Gauchetiere, Montréal Québec H5A 1K6, \\ Canada.boutayeb@emt.inrs.ca. Fax: (514)-875-0344
}

\begin{abstract}
A new miniature microstrip four-pole bandpass filter using a two-layer structure with quarter-wavelength resonators and open stub inverters is proposed. The coupling between the resonators in the two layers is achieved via a slot etched on a common ground plane located between the layers. The open stub inverters are used in order to increase the selectivity of the filter and to avoid connection to ground plane that is generally required with quarter-wavelength resonators of bandpass filter. A four-pole bandpass filter with elliptic-type performance is designed and tested at $2.45 \mathrm{GHz}$.
\end{abstract}

Key words: Bandpass filter; K-inverter; Miniaturization; Microstrip; Multilayered Structures 


\section{Introduction}

In wireless communication systems, bandpass filters with small size, low insertion loss and high selectivity in the pass-band are required. For designing compact microstrip filters, several structures have been proposed such as the hairpin resonator, the hairpin-comb resonator or the square open loop resonator [1-3]. However, the proposed filters are generally too large to be used for wireless handset communication systems, where engineers are always looking for smaller microwave components.

A method to reduce the size of these filters consists on using multilayered structures. In [4-6], multilayered bandpass filters, based on resonators with different geometries (square-loop, hairpin or stepped impedance resonators), have been proposed.

Another way for size reduction has been proposed in [7]. This technique uses an open stub inverter between quarter-wavelength resonators, which results in compact design as well as low loss. The open stub inverters allow also increasing the filter selectivity and avoiding the shorting to ground that is generally required with quarter-wavelength resonators of bandpass filters.

This letter presents a new two-layer microstrip four-pole bandpass filter that uses quarterwavelength resonators with open stub inverters. The two-layer configuration allows reducing the size of a conventional single layer microstrip filter by $50 \%$ without increasing the insertion loss.

The remainder of this paper is organized as follows. In Section II, the design technique for the quarter-wavelength resonators with open stub inverters in multilayer topology is presented. To validate the proposed approach and scheme, experimental results for a filter of elliptic-type performance and operating at $2.45 \mathrm{GHz}$ are presented in Section III. Finally, concluding remarks are given in Section IV.

\section{Filter Design}

In this section, we present the different elements that constitute the four-pole bandpass filter. The proposed filter is composed of two two-pole filters. These two-pole filters consist of two quarter-wavelength resonators and a tapped open stub. Figure 1 shows the layout of the two-pole filter and its equivalent circuit. $Z_{1}$ and $Z_{2}$ are the characteristic impedances of the resonators and the open stub inverter, respectively. The tapped open stub works as a K-inverter and also introduces an attenuation pole. The attenuation pole can be located at upper or lower side of the passband by adjusting the open stub length. The inverter value is fixed by the characteristic 
impedance $Z_{2}$ and the length of the stub. The length variation of the tapped open stub allows controlling the notch frequency in the stop-band [7]. When the characteristic impedance $Z_{2}$ of the stub increases, the attenuation pole is located closer to the pass-band edge.

Figure 2 shows the complete proposed four-pole filter structure and the different parameters that were optimized. This structure consists of two two-pole filters etched on two different layers and of a common ground plane, where a slot is introduced to ensure the coupling between the two parts of the structure. $c_{1}$ and $c_{2}$ denote the lengths of the stubs in the upper and lower layers, respectively. $g$ is the coupling gap size and $d x$ and $d y$ are the dimensions of the slot. $w_{1}, w_{2}$ and $w_{3}$ are the line widths or the J-inverters, the resonators, and the stubs, respectively, whereas $a, b_{1}$, and $b_{2}$ denote the line sections lengths of the resonators as shown in Fig. 2.

One can note that the proposed structure allows a reduction by $50 \%$ compared to the four-pole bandpass filter configuration proposed in [7].

\section{Experimental results}

To validate our approach, a bandpass filter was designed and fabricated to operate at $2.45 \mathrm{GHz}$. The filter was fabricated on Rogers RO4003 substrates with relative dielectric constant of 3.38 and a thickness of $0.508 \mathrm{~mm}$. The dimensions of the two-pole filters and of the slot were optimized by using a full-wave electromagnetic simulator (IE3D [8]). The obtained optimized values of the parameters of the structure (see Fig. 2) are the followings: $a=13.502 \mathrm{~mm}, b_{1}=12.2$ $\mathrm{mm}, b_{2}=12.1 \mathrm{~mm}, c_{1}=19.3148 \mathrm{~mm}, c_{2}=18.5137 \mathrm{~mm}, g=0.1598 \mathrm{~mm}, d x=2.357 \mathrm{~mm}$, and $d y$ $=1.216 \mathrm{~mm}$. For these parameters values, the characteristic impedances of the microstrip line sections of the resonators and of the open stub are $Z_{1}=49.2 \Omega$ and $Z_{2}=61.4 \Omega$, respectively.

Figure 3 shows the photograph of the fabricated prototype. In this figure, only the two quarterwavelength resonators on the top layer are visible. The size of the fabricated filter is $18.71 \mathrm{~mm} \times$ $20.51 \mathrm{~mm}$.

Figure 4 shows the measured and simulated S-parameters of the filter. From these curves, it can be observed that a good agreement is achieved between theoretical and experimental results. The measured insertion loss within the pass-band is about $1.05 \mathrm{~dB}$, which is higher than the simulated one $(0.05 \mathrm{~dB})$. This is due to the losses in the SMA connectors, the dielectric, and the conductor that were not taken into account in simulations. However, the measured insertion loss is still low. Within the pass-band, the reflection coefficient is less than $-12.5 \mathrm{~dB}$, and the filter presents a high 
selectivity. A fractional bandwidth of $3.279 \%$ around $2.45 \mathrm{GHz}$ is achieved. With such features, the proposed miniaturized filter is suitable for wireless communication systems.

\section{Conclusion}

A new miniaturized four-pole bandpass filter using a two-layer structure with microstrip quarterwavelength resonators and open stub inverters has been presented. In the proposed design, the coupling between resonators in the two layers is achieved through an aperture etched in a common ground. Based on this architecture, the filter has a size reduced by $50 \%$ compared to conventional single layer microstrip filters. Furthermore, the proposed design technique is simple. Experimental results for a bandpass filter operating at $2.45 \mathrm{GHz}$ have been presented. The obtained results have shown good performances in terms of low insertion loss and high selectivity in the pass-band, which is suitable for wireless handset communication systems.

\section{Acknowledgement}

This work was supported in part by National Science Engineering Research Council of Canada (NSERC). 


\section{REFERENCES}

1. J. Hong and M. J. Lancaster, Cross-coupled microstrip square open-loop resonators for cross-coupled planar microwave filter, IEEE Trans Microwave Theory Tech 44 (1996), 2099- 2109.

2. J. T. Kuo, M. J. Maa, and p. H. Lu, A microstrip elliptic function filter with compact miniaturized hairpin resonators, IEEE Microwave and Guided Wave Lett 10 (2000), 94-95.

3. J. T. Kuo and E. Shih, Microstrip stepped impedance resonator bandpass filter with an extended optimal rejection bandwidth, IEEE Trans Microwave Theory Tech 51 (2003), 1554-1559.

4. J. S. Hong and M. J. Lancaster, Aperture-Coupled Microstrip Open-Loop Resonators and their Applications to the Design of Novel Microstrip Bandpass Filter, IEEE Trans Microwave Theory Tech 47 (1999), 1848-1855.

5. A. Djaiz and T. A. Denidni, A Reduced-Size Two-Layer Bandpass Filter, Microwave Opt Technol Lett 44 (2005), 512-515.

6. A. Djaiz and T. A. Denidni, A new Two-Layer Bandpass Filter using Stepped impedance hairpin resonators for wireless applications, IEEE MTT-S Int Microwave Symposium 3 (2005), 1487-1490.

7. J. R. Lee, J. H. Cho, and S.W. Yun, New Compact Bandpass Filter Using Microstrip $\lambda / 4$ Resonators with Open Stub Inverter, IEEE Microwave and Guided wave Lett 10 (2000), $526-527$.

8. IE3D 8. 2, Zeland Software, Inc Fremont, CA, USA. 


\section{Figure Captions:}

FIG. 1: Two-pole bandpass filter: (a) layout, (b) equivalent circuit.

FIG. 2: Layout of the bandpass filter, (a) upper layer, (b) common ground, (c) lower layer.

FIG. 3: Photograph of the fabricated Bandpass filter.

FIG. 4: Simulated and measured $S_{11}$ and $S_{21}$ parameters of the compact filter. 
FIG. 1

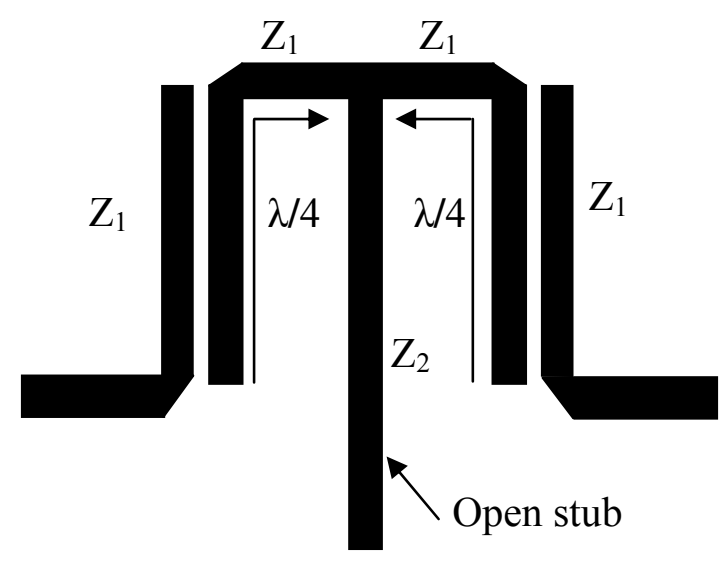

(a)

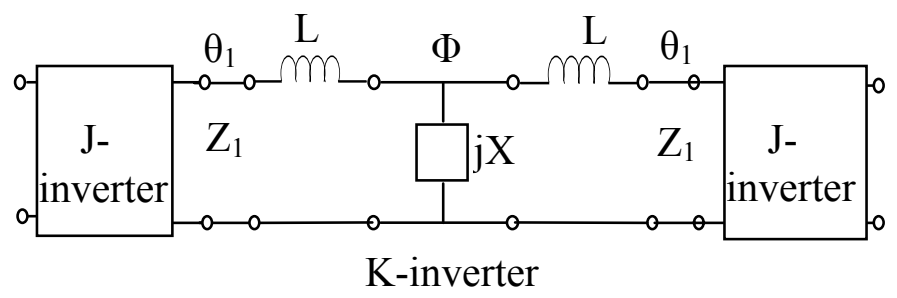


FIG.2

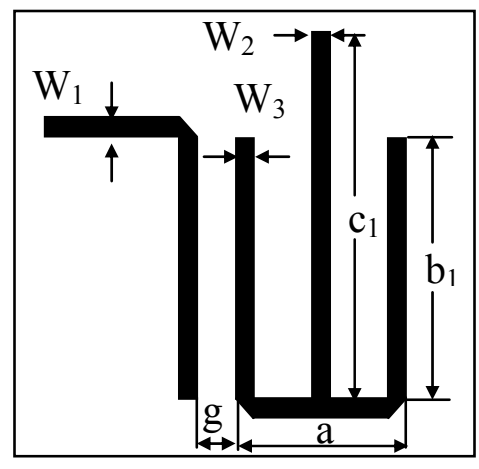

(a)

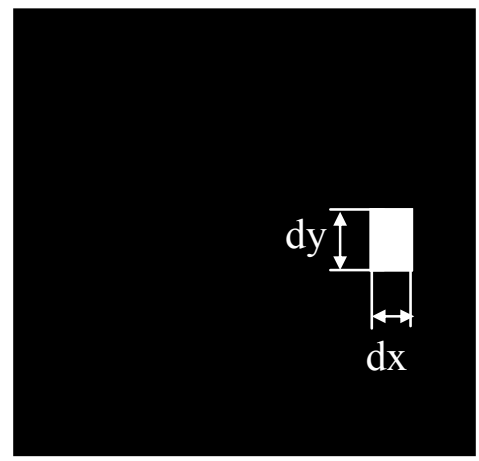

(b)

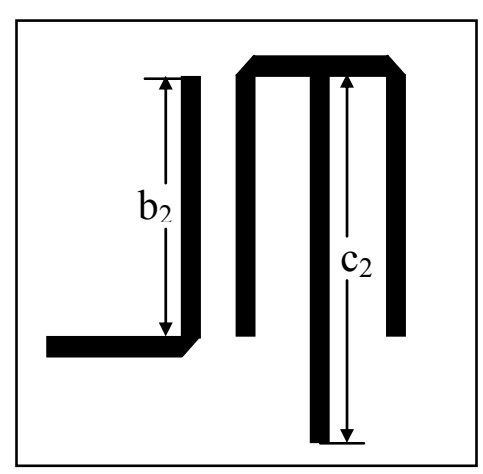

(c) 
FIG.3

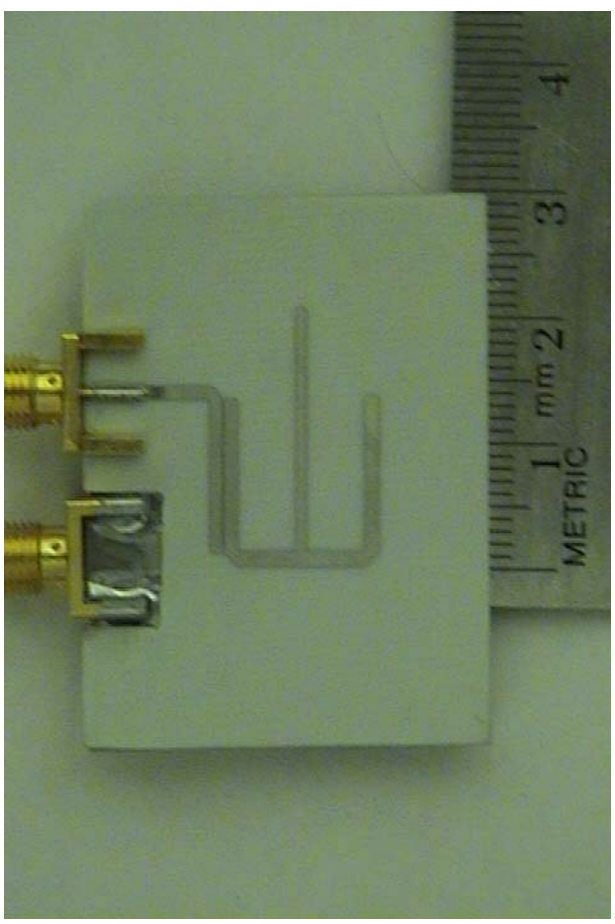


FIG. 4

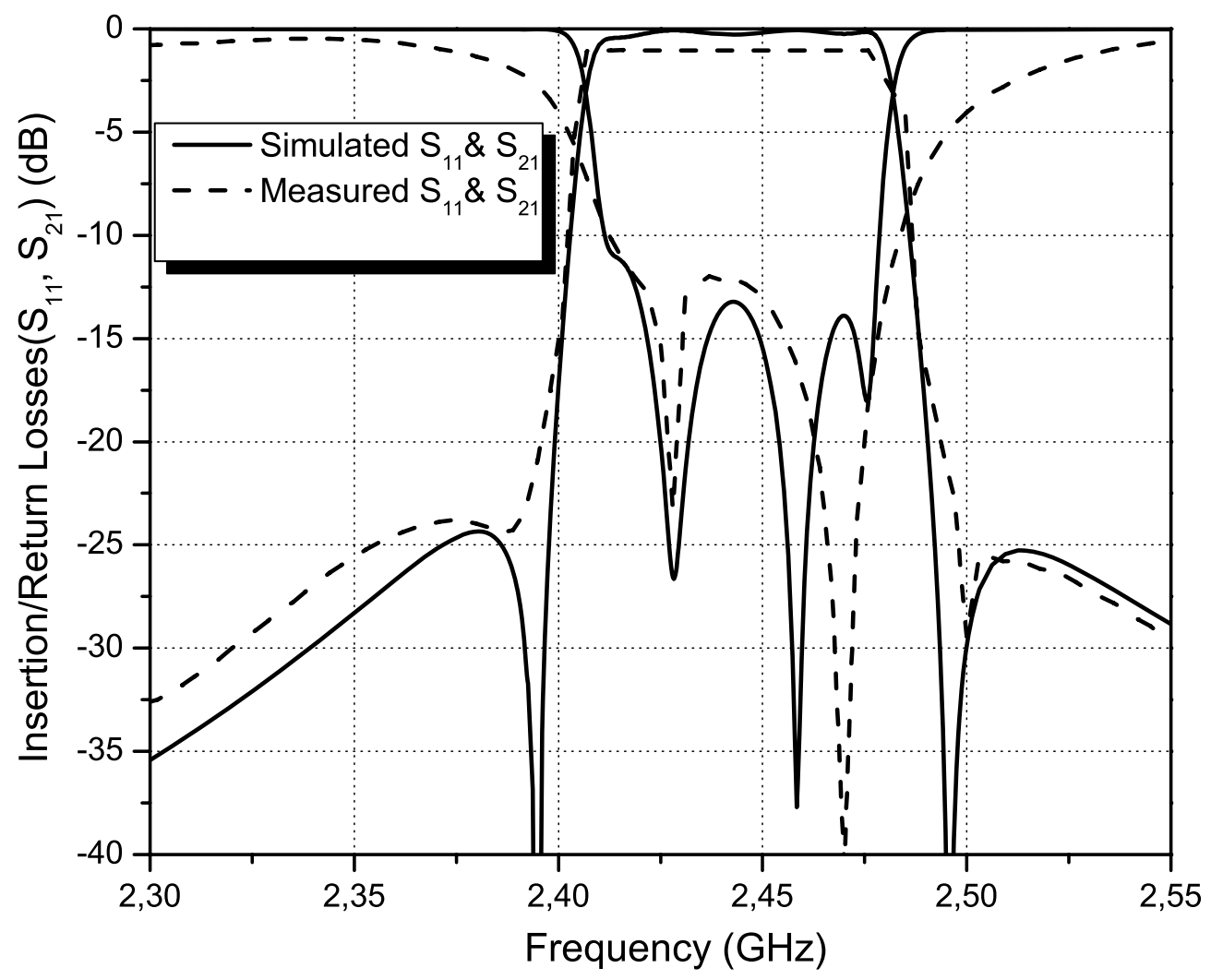

